\title{
The Fractal study in complex product self-similar form
}

\author{
Li Gang1, 2,a , Yu Suihuai1,b, He Weiping ${ }^{1, b}$ \\ ${ }^{1}$ Instiute of Industrial Design Northwestern Polytechnical University Xi'an, China. \\ ${ }^{2}$ The industrial design Kunming University of Science and Technology,Kunming,china \\ afractal@139.com, bysuihuai@vip.sina.com, cweiping@nwpu.edu.cn
}

\begin{abstract}
Keywords: complex system, sierpinski triangle, product modeling , self similarity, dissipative structure
\end{abstract}

Abstract. The paper analyzes Sierpinski self-similar feature of fractal, The paper also suggests product form modular design model and define multiple factors on complex product modeling through the interrelation between Similar Mapping and Fixed Point. The paper verifies the self-similar evolution method where the product gene feature can act as initial condition of complex product modeling by studying changing method for Sierpinski self-similar multiple form with combination of application of self-similar principle to complex product modeling design.

\section{Introduction}

The complex system is widely spread among the nature as well as the human society, which is made up of natural system, artificial system and the superimposed system which contains the former two. Xuesen Qian,Wolf F,M suggested the complex giant system, which studied profoundly the systematic character of the complexity for products. Its feature is not only the simple system and the stochastic system, but the composite system which has multilevel as well as multivariate effects. [1,2]

There are kinds of interrelated influences among the subsystem, between the subsystem and the overall system, on top of that, the system and the environment. It has a certain nonlinear system with order and randomness in it, and the coevolution can be found on them. The nonlinear characteristics affects diversification significantly through the process of complex design on product modeling. The fractal is a kind of typical complex system, which acts as the geometric language for nonlinear motion, and which has important research value in the formation and the evolution of complex systems. The research of self similarity for fractal as well as the multiscale structure plays an active role in complex product form design.[3]

As for the complex product form, there is a gradually change in internal system as well as the external environment, and there will be gradual possible issue which leads to the mutation for complex product overall system. Consequently, the application of fractal model has effects within a certain range, so it is hard to have a long-term forecast. The paper studies the self similarity behavior in a short-term and within a certain range on complex product form by employing the generating features of typical fractal model Sierpinski triangle and the self similarity, which combines with the genetic characteristics on complex product modeling as well as the multiple factors.

From the fractal process we can find out the fact that the genetic characteristic for complex product is only one of the characteristic of the fractal behavior. The influences of multifactor will result in great changes towards overall system, and the change of multifactor is always complex, which leads to the evolution of complex product itself as well as the form of product family. As the complex system, the complex product is uncertain, dynamic, stable and interoperable. Consequently, we ought to take the effect of multifactor into account when we make a decision towards the complex product.

\section{Realted works}

Baogui $\mathrm{Wu}$ and other researchers suggest the general definition of complex product, they study basic methods on multidisciplinary optimization modeling of complex product, they present an issue 
that multifactor influences on complex product is very important[4]. Renmiao Zhu and other researchers simulates product design process employing fractal theory and combining with matrix on the complexity as well as the dynamic feature on complex electromechanical product design process in that they employs the mega-idea of fractal.[5,6,7] Puligaojin builds up dissipative structure, they study open structure feature, and the nonlinear interaction within the system far from equilibrium state.[8] The open system of dissipative structure plays an important role in dealing with external environment effects, and furthermore, the system far from equilibrium will easily lead to the system evolution. Consequently, the non-equilibrium state is a kind of important effect on the evolution of complex product modeling process. Shuying Wang studies that the imitated shift mapping of power system has the same effect with self mapping of shift invariant set through describing imitated shift mapping of Sierpinski triangle, which has a certain role in genetic characteristic in complex product family.[ Shenbing Ni suggests the self similarity as well as the evaluation model of fractal supply chain. But the previous research seldom concerned about evolution process and gene mechanism for the complexity of product shape. $[9,10,11]$

\section{The proposed scheme}

The paper defines the influence factors on complex product modeling, which include the factors which will possibly affect such as Form $(F)$, Color $(C)$, Material $(M)$, HMI $(H)$, Struture relationship $(S)$, User Demand Study $(U)$ and the Product Feature $(D)$.

Generally, complex product modeling is:

$$
A=f(F, D, U, C, S, M)
$$

Consequently, the different roles made from gradual changes of those factors in complex product modeling design are the important feature of diversified modeling of complex product. The products' mapping relation according to their different roles in the whole is also different. Mapping that lays emphasis on Form is:

$$
\begin{gathered}
A=f\left(F^{\prime}, F\right) \\
F^{\prime}=f(F, D, \quad U, C, S, M)
\end{gathered}
$$

As for the modern industrial design, the evolution process and system of product modeling are very complex, we must take inner environment, outer environment, genetic environment, systematic environment and the brand genetic characteristic. Lots of research indicates that one of the effective methods to form product family are modular design, series design, universal design and the standardized design. The product module has the independent feature and standard input and output components, it is widely used, on top of that, it can effectively reduce development costs, increase development speed, and also it can enhance the competitiveness of enterprises.

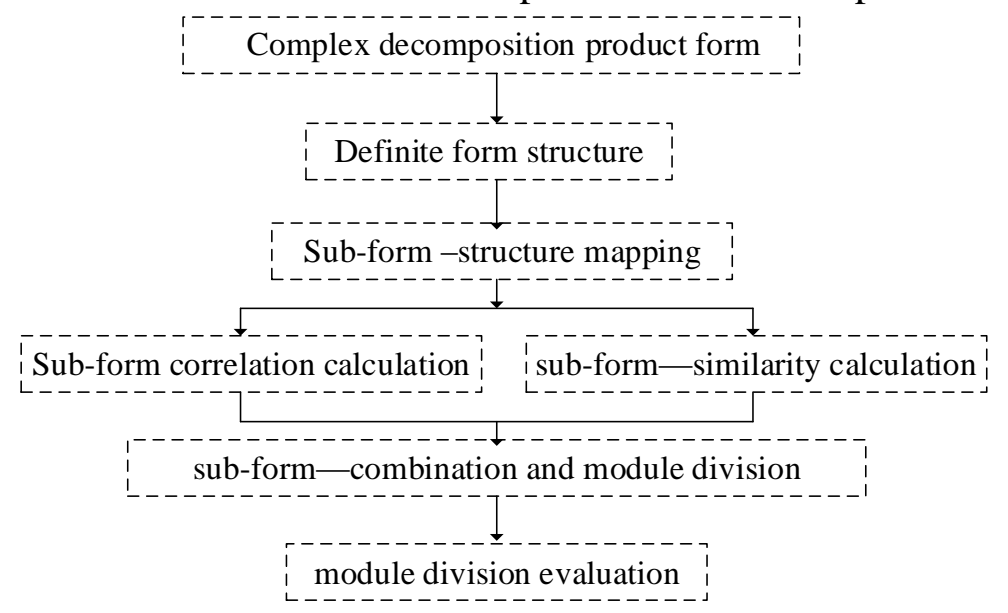

Fig. 1: Complex decomposition product form and mapping process 
We can form series function module combination through the combination of different functions and same functions within a certain range. The different functional module combination can form different series products, which can satisfy diverse usage requirements from many family users.

. Fig 1 is Complex decomposition product form and mapping process. It determines sub-form structure through form decomposition, and we can have morphological evolution by correlation and similarity calculation when those sub-form structure acts as product family gene. According to chart 2 , decomposition of sub-form can act as self-similar form gene, which can make sure that post-evolution complex product form can have the same feature of self-similarity with the previous product form, and it is not only applied to product family design in the same enterprise, but also to the similar products industry.

The evolution for self similarity of complex product family form is in fig 2 . There it chooses product form gene $A$ as product gene, it can make different products, 1, 2 and 3 through different function module 1, function module 2 and function module 3 , and they have the same form self-similar gene A.

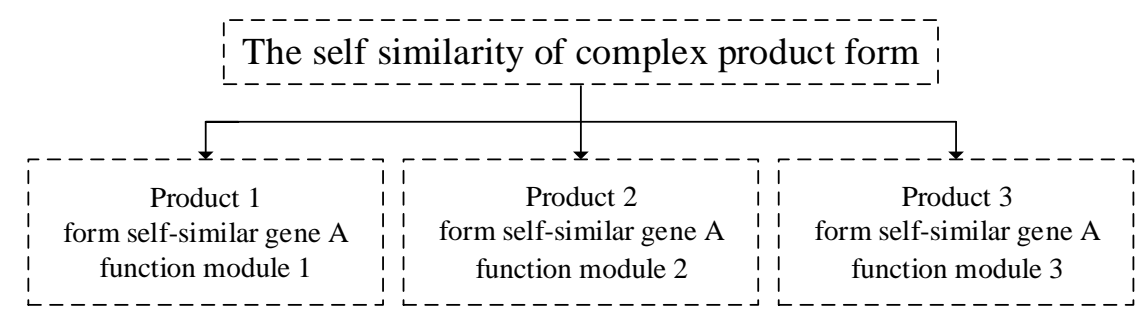

Fig. 2 The self similarity of complex product form

\section{System model}

Suppose that complex product form is set $A$, and define $A$ the compact set. Complex product form gene $S_{1}, S_{2}$ and $S_{3}$ as self-similar. Suppose that $S: A^{n} \rightarrow A^{n}$ as self-similar, and if $\exists c \in(0,1)$ can make $|S(x)-S(y)|=c|x-y|, \forall x, y \in A^{n}$, and in that $\mathrm{c}$ is the compression ratio of $S$, self-similar $S_{1}$, the result of same time self-similarity of $S_{2}, S_{3}, \cdots, S_{k}$ is $\bigcup_{i=1}^{k} S_{i}$, it can determine $\left(\bigcup_{i=1}^{k} S_{i}\right)(x)=\bigcup_{i=1}^{k} S_{i}\left\{S_{i}(x)\right\}, \forall x \in R^{n}$, we can have result that $A=S(A)=\bigcup_{i=1}^{k} S_{i}(A)$, 可 知 $A=\$ S \quad S \cup{ }_{2} S C \quad \bigcup_{3} \quad S$. That is, there is self-similarity in the complex product modeling $A$ set. So, its rule can be applied to complex product family design.

Sierpinski structural property of related graphs could solve too many complex problems. Suppose that product form gene is top $S_{1}$, top $S_{2}$ and Top $S_{3}$. Rule of Decomposition of complex product form is the chart 2, that is, Complex decomposition product form and mapping process, while its evolution of self-similarity is the chart2, The evolution for self similarity of complex product form.

Set up an array by Sierpinski triangle formula . it is the rule of form evolution. . the third dimension has two layers, the first is $\mathrm{x}$, the second is $\mathrm{y}$. The system initial influence factor $x=[0.5,1,0.5]$, $y=[0,0.5,0.5]$, the iteration time is 5 .

Standard Sierpinski triangle is the similar mapping which has the compression ratio $\frac{1}{2}$. In order to increase form diversity, we can adjust the rule of Sierpinski triangle. Suppose that product form gene is the top $S_{1}=\frac{1}{3}$, and then it can have form evolution. 


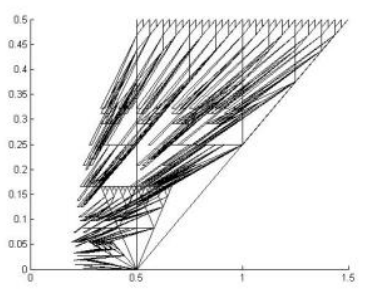

Fig. 3product form 1

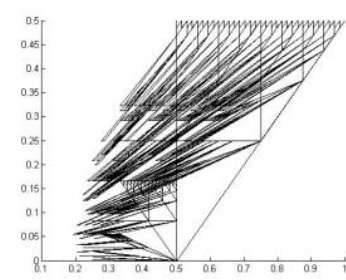

Fig. 4:product form2

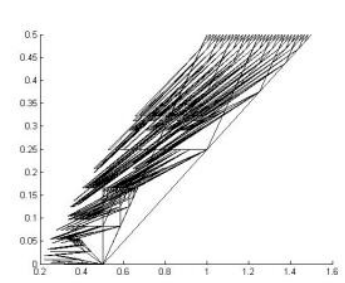

Fig. 5:product form3

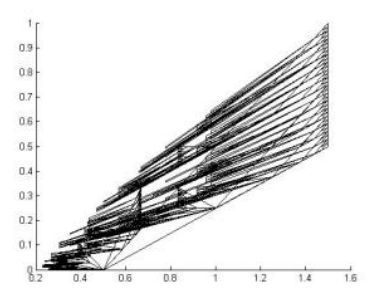

Fig. 6: product form 4

In that the chart 3 is product form of the system initial influence factor that is, $x=[0.5,1,0.5]$, $y=[0,0.5,0.5]$. Here we can make three form chart $, 5,6$ and 7 through altering system initial influence factor.

Chart 4 is the product form of system initial influence factor $x_{1}=[0.5,1,1.5], \quad y_{1}=[0,0.5,0.5]$ Chart 5 is the product form of system initial influence factor $x_{2}=[0.5,1.5,1.5], \quad y_{2}=[0,1,0.5]$ Chart 6 is the product form of system initial influence factor $x_{3}=[1,1.5,2.5], \quad y_{3}=[0.5,1,0.5]$ Under the circumstances that product form production rules as well as the factor that product gene top $S_{1}, S_{2}$ and $S_{3}$ are the same with before, it can make product family which has self-similar fractal.

\section{Conclusion}

It suggests modular model of complex product form through studying multiple factors on complex product form, and this can analyze rule of product modeling evolution. And on top of that, it shows the product form gene combining with fractal self-similar principle, and it builds up complex product form model by employing form rule of Sierpinski triangle, and furthermore, it can form series product family form, and then it can produce multiple self-similar module under the same form rule with same product gene, which can help the designers to design complex product form through fractal self-similar principle. The next work will be the complex product form evolution according to the existing model under too much more factor.

\section{References}

[1] Wolf F.M.Mela-Analysis: QualitativeMethods for Research Synthesis. Sage. 1986

[2] Hedges, L,Olkin. I.Statistical Methods for Meta-Analysis.Academic Press. 1985

[3] Mandelbrot B B. The fractal geometry of nature[M]. San Fransisco: Freeman W H, 1982.5-47.

[4] Bay N,Nielsen M S/Mechanical processing of AG/BSCCO High temperature superconductor tape[J]journal of Materials Processing Technology,2004,151:18-26

[5] Renmiao Zhu.Product design process modeling based on fractal thought[J].Computer integrated Manufacturing Systems. 2011,12:2573-2582.(in chinese)

[6] OSTROSI E,FERNEY M,GARRO O.A fractal-based approach for concurrent engineering[J].Concurrent engineering,2003,11(4):249-265

[7] KUSIAK A,WANG J R,Decomposition of the design process[J].Journal of Mechanical Design,1993,115(4):687-696

[8] Prigogine I, Time, Structure and Fluctuation[J].Science,1978,14(5):438-452

[9] Ryu K,JUNG M. Goal-orientation mechanism in a fractal manufacturing system[J]. International Journal of Production Research.2004,42(11): 2207-2225

[10] RYU K,SON Y,JUNG M.Modeling and specifications of dynamic agents in fractal manufactruing systems [J]. Computers in industry,2003,52(2):161-182

[11] SAAD S M,LASSILA A M,Layout design in fractal organization[J]. international journal of Production Research,2004,42(17):3529-3550 